\title{
Molecular analysis of an odorant-binding protein gene in two sympatric species of Lutzomyia longipalpis s.l.
}

\author{
Ana Karina Kerche Dias ${ }^{1}$, Luiz Guilherme Soares da Rocha Bauzer1/+, \\ Denise Borges dos Santos Dias, ${ }^{1,2}$, Alexandre Afranio Peixoto ${ }^{1,3,+}$
}

\begin{abstract}
${ }^{1}$ Laboratório de Biologia Molecular de Insetos, Instituto Oswaldo Cruz-Fiocruz, Rio de Janeiro, RJ, Brasil ${ }^{2}$ Departamento de Bioquímica, Instituto de Biologia Roberto Alcântara Gomes, Universidade do Estado do Rio de Janeiro, Rio de Janeiro, RJ, Brasil ${ }^{3}$ Instituto Nacional de Ciência e Tecnologia em Entomologia Molecular, Rio de Janeiro, RJ, Brasil
\end{abstract}

Lutzomyia longipalpis s.l. is the main vector of American visceral leishmaniasis (AVL) and occurs as a species complex. DNA samples from two Brazilian sympatric species that differ in pheromone and courtship song production were used to analyse molecular polymorphisms in an odorant-binding protein (obp29) gene. OBPs are proteins related to olfaction and are involved in activities fundamental to survival, such as foraging, mating and choice of oviposition site. In this study, the marker obp29 was found to be highly polymorphic in Lu. longipalpis s.l., with no fixed differences observed between the two species. A pairwise fixation index test indicated a moderate level of genetic differentiation between the samples analysed.

Key words: Lutzomyia longipalpis s.l. - odorant-binding proteins - leishmaniasis

The sandfly Lutzomyia longipalpis s.l. is the main vector of American visceral leishmaniasis (AVL) and occurs as a complex of cryptic species (Bauzer et al. 2007, Araki et al. 2009). A number of integrative studies using different approaches, such as molecular polymorphisms (Bauzer et al. 2002a, b, Bottecchia et al. 2004, Araki et al. 2009, Lins et al. 2012), microsatellites (Maingon et al. 2003), pheromones (Hamilton et al. 1999a, b, Souza et al. 2004) and courtship song analysis, have revealed the existence of at least five species in Brazil (Souza et al. 2002, Araki et al. 2009). Lu. longipalpis s.l. males exhibit morphological polymorphism with regard to the number of pale abdominal tergal spots and can present just one pair of pale spots on the fourth abdominal tergite (1S phenotype) or two pairs of pale spots, one on the third and the other on the fourth abdominal tergite ( $2 \mathrm{~S}$ phenotype). Intermediate phenotypes (a small spot on the 3rd tergite in addition to the spot on the 4th tergite) are observed in high frequencies in some localities, indicating an intraspecific polymorphism (Ward et al. 1988). In contrast, intermediate forms are rare or nonexistent in localities where two Lu. longipalpis s.l. cryptic species occur in sympatry (Bauzer et al. 2002a, Araki et al. 2009). These tergal spots contain pheromone glands that are associated with Lu. longipalpis sexual communication (Lane et al. 1985). In the Brazilian locality of Sobral, state of Ceará, a sympatric species identified by

doi: 10.1590/0074-0276130449

Financial support: HHMI, FIOCRUZ, CAPES

${ }^{\dagger}$ In memoriam

+ Corresponding author: lbauzer@ioc.fiocruz.br

Received 13 September 2013

Accepted 29 November 2013 the $2 \mathrm{~S}$ phenotype was shown to produce the cembrene-1 $\left(\mathrm{C}_{20}\right)$-type pheromone, whereas a species associated with the $1 \mathrm{~S}$ phenotype was shown to produce the 9-methylgermacrene- $\mathrm{B}\left(\mathrm{C}_{16}\right)$-type pheromone (Lane et al. 1985, Hamilton et al. 1999a, b).

Proteins related to olfaction are involved in activities that are fundamental to survival, such as foraging, courtship, mating and choice of oviposition sites (Hallem $\&$ Carlson 2004). Among these molecules, odorant-binding proteins (OBPs) have been described as important components in the recognition of odours (Hekmat-Scafe et al. 2002) and several OBPs from different insect species have been cloned and sequenced (Xu et al. 2003, Zhou et al. 2004, 2008). Furthermore, an increasing number of OBPs have been identified in several nonsensorial tissues, such as salivary glands (Abdeladhim et al. 2012), head and body (Li et al. 2005, González-Caballero et al. 2013), reproductive organs (Azevedo et al. 2012), fat bodies and seminal fluid (Liu et al. 2010, Sirot et al. 2011). The wide distribution of these proteins in the organism suggests that they can perform several other physiological functions (Pelosi et al. 2006, Pelletier \& Leal 2009). Phylogenetic analyses and the distribution and orientation of OBP genes in insect genomes have revealed evidence of a complex series of duplication and rearrangement events, facts that suggest that this gene family evolves rapidly (Hekmat-Scafe et al. 2002, Vieira et al. 2007) and are therefore potentially good markers for use in population genetic studies.

In this study, we analysed polymorphisms in the obp29 gene to characterise the molecular variation of sympatric species of Lu. longipalpis s.l. and to hypothesise a possible role of the encoded OBPs in the reproductive isolation and adaptation of this important insect vector.

The genomic DNA from samples collected in the Brazilian locality of Sobral ( $\left.3^{\circ} 41^{\prime} \mathrm{S} 40^{\circ} 20^{\prime} \mathrm{W}\right)$ was the same as that used by Bauzer et al. (2002a.) Genes that were isolated from Lu. longipalpis s.l. and searched 
for using the Antennae EST database developed in our laboratory (Dias 2008) directed the choice of specific oligonucleotide primers for the obp29 gene. Polymerase chain reaction (PCR) reactions were performed according to the Go Taq DNA Polymerase instructions manual (Promega) using a thermocycler (GeneAmp PCR System 9700/Applied Biosystems) with forward (5'-GGGAGTGAGAAAAATGAGATCAAA-3') and reverse (5'-ACTTGACATTGCTTTTCTGTGCAGG-3') OBP29 primers. The following programme was used: 30 cycles of $95^{\circ} \mathrm{C}$ for $30 \mathrm{sec}, 60^{\circ} \mathrm{C}$ for $30 \mathrm{sec}$ and $72^{\circ} \mathrm{C}$ for $1 \mathrm{~min}$. The obtained fragments were purified using the QIAquick PCR Purification kit (QIAGEN) and cloned using the pGEM-T Vector kit (Promega). Plasmid DNA was isolated using the mini-preparation alkaline lysis method (Sambrook \& Russel 2001) in 96-well MicroWell Plates and the DNA was cleaned using filter plates (Multiscreen-Millipore). Sequencing of the cloned fragments was carried out using the ABI Prism Big Dye Terminator Cycle Sequencing Ready Reaction v.3.1 kit (Applied Biosystems) and the ABI 3730 Sequencer by the Program on Technological Development of Health (Fiocruz, Rio de Janeiro, Brazil) sequencing service (Otto et al. 2008). The obtained sequences were edited using the Bioedit v.7.0.5 programme (Hall 1999) and an alignment was performed using the CLUSTALW full multiple alignment option within the BioEdit software. Different measures of genetic polymorphism were calculated using DnaSP v.5 (Librado \& Rozas 2009), including the number of segregating sites (S), the nucleotide diversity pi $(\pi)$ which is the average number of nucleotide substitutions per site between any two sequences and the parameter theta $(\theta)$, which is estimated from the number of segregating sites. The estimated $\pi$ and $\theta$ parameters were used to perform Tajima's D test of neutrality (Tajima 1989). The Hudson, Kreitman \& Aguadé neutrality test (HKA) was performed using the period gene (Bauzer et al. 2002a) as the second locus. Both neutrality tests were executed using the DnaSP v.5 software (Librado \& Rozas 2009). A population structure analysis was conducted by separating two groups of sequences based on the abdominal spot phenotype (1S or $2 \mathrm{~S}$ ). The population subdivision was calculated using the programme ProSeq3.0 (Filatov 2002) and the significance of the pairwise fixation index test was estimated by a permutation approach. The sequences were submitted to GenBank (accessions KF669570-KF669605).

A 685-bp opb29 fragment encompassing the coding region and part of the 3' UTR was obtained. A total of 18 consensus sequences were analysed from the Sobral $1 \mathrm{~S}$ and $2 \mathrm{~S}$ populations. Of the 685 analysed nucleotides sites, $93(13.6 \%)$ were variable and the nucleotide diversi-
SOB1S_ind03_conA SOB1S ind 14 con SOB1S_ind03_conB SOB1S ${ }^{-}$ind 04 COnA SOB1S ind 04 COnB SOB1S_ind05_conA SOB1S ind 05 ConB SOB1S ind 6 COnA SOB1S_ind06_conB SOB1S ind $07^{-}$con SOB1S ind 08 con SOB1S_ind09-con SOB1S_ind11_conA SOB1S ind 11 con $B$ SOB1S_ind12_conA SOB1S_ind12_conB SOB1S ind13 ${ }^{-}$ConA SOB1S_ind13_con B SOB2S_ind03_conA SOB2S ind12 ConA SOB2S ind03-conB SOB2S_ind04_conA SOB2S_ind05_conA SOB2S ind 5 ConB SOB2S_ind06_conA SOB2S_ind06_conB SOB2S ind $07^{-}$ConA SOB2S ind $7^{-}$ConB SOB2S_ind08_conA SOB2S ind 08 Con $B$ SOB2S_ind09-COnA SOB2S ind09-conB SOB2S ind 10 conA SOB2S_ind10_conB SOB2S ${ }^{-}$ind $11^{-}$ConA SOB2S_ind11_conB
000000000011111111122222222222222233333333333444444444444444444444455555555555556666666666666 124456678811124457700111233667788901145667899001122233344466777789902233455667791233355677778 080354735858942642758124958281409113872485127392514706915839235843920325736584759978916304793 CCCCTCAGCCGTGTATGAACGCAAGAAATTGGCATTGAGCCTGCGCGTCACACCCGCCGGATGGCCCAGAGACTCCCTCACCTCTCCTTTTTT

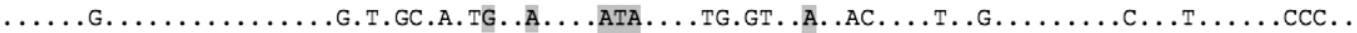

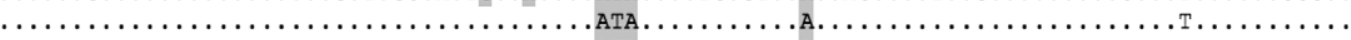

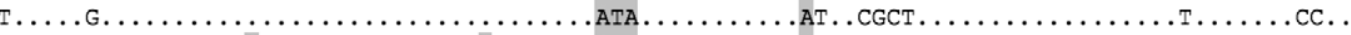

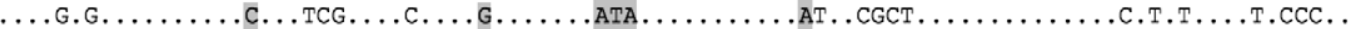

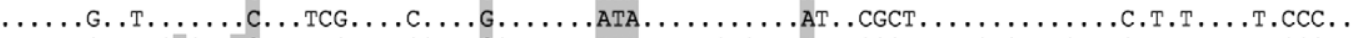

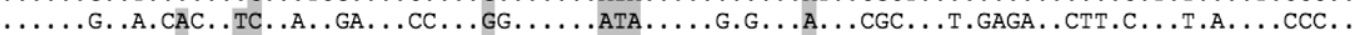

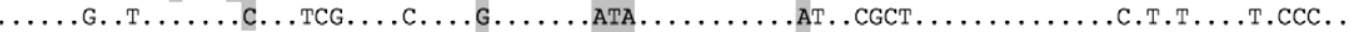
$\ldots \ldots \ldots \ldots \ldots \ldots \ldots$. T. . . . . . . . ATA. . . . . . . . . . . . . . . . . . . . .

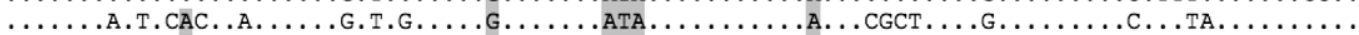

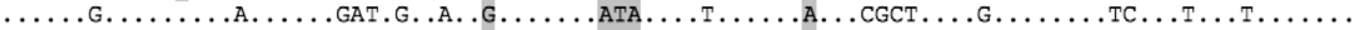

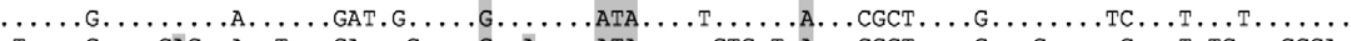

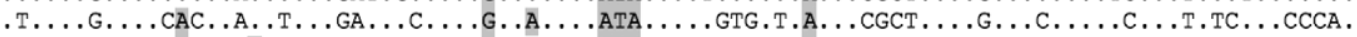

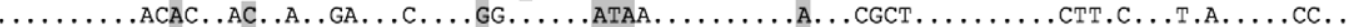

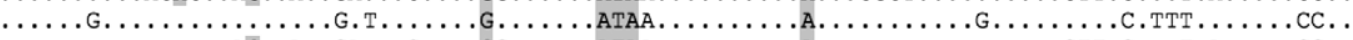

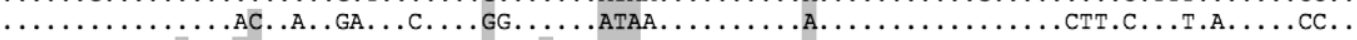

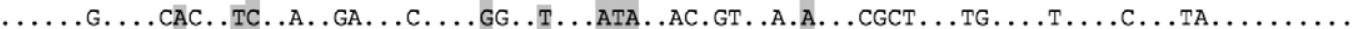

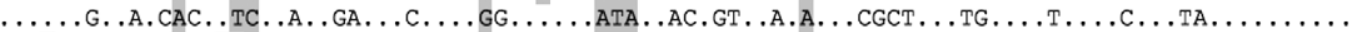

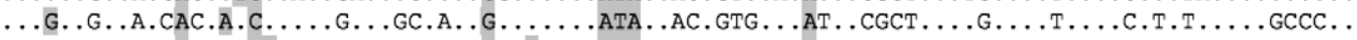

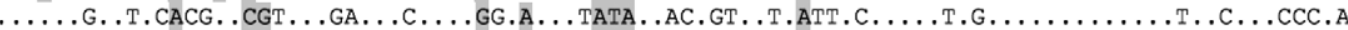

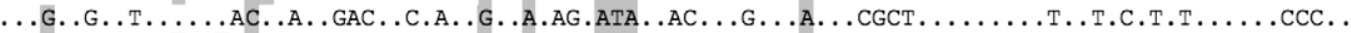

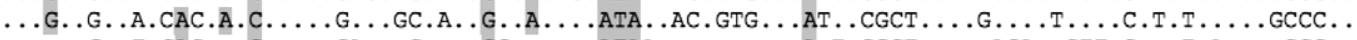

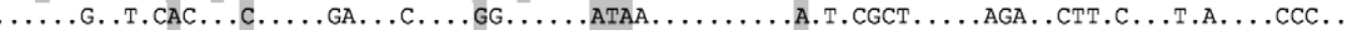

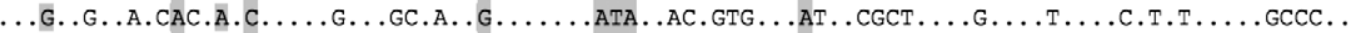

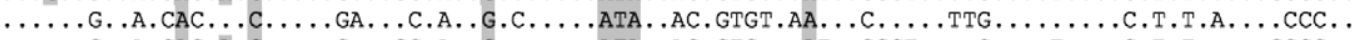

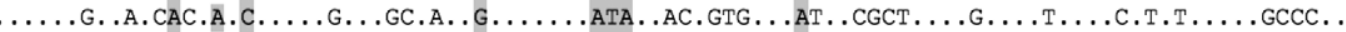

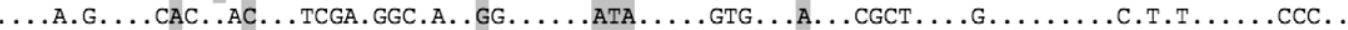

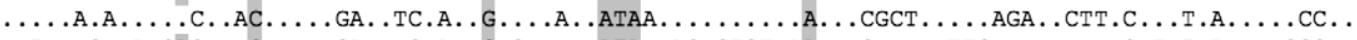

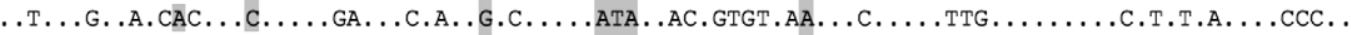

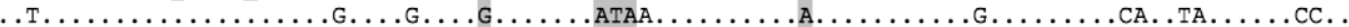

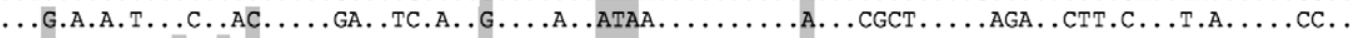

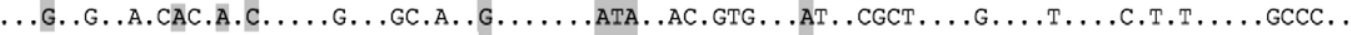

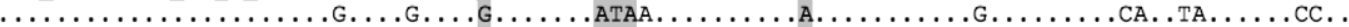

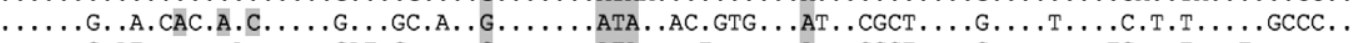

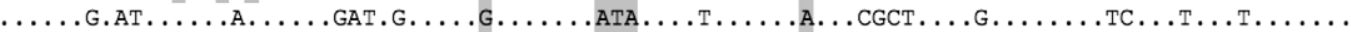

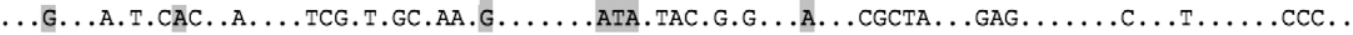

Alignment of the variable sites in the Lutzomyia longipalpis s.l. odorant-binding protein 29 haplotypic sequences. Dots indicate nucleotides shared with the first sequence. Positions of the polymorphic sites are indicated on the top of the figure. Non synonymous sites are shown in gray. 
ty estimation was similar for the two species analysed $(\pi$ $=0.035$ for Sobral $1 \mathrm{~S}$ and $\pi=0.0331$ for Sobral $2 \mathrm{~S}$ ). Similarly, the parameter $\theta$ was found to be essentially the same for the two species $(0.0310$ and 0.0309 for Sobral $1 \mathrm{~S}$ and Sobral 2S, respectively). Figure shows an alignment of all the polymorphic sites observed in the analysed opb29 fragment. Twenty three polymorphic sites were exclusive to Sobral 1S, 24 were exclusive to Sobral $2 \mathrm{~S}$ and 54 were shared between the species; non-synonymous mutations were found at 12 sites (6 exclusive to S1S, 3 exclusive to S2S and 3 shared). A moderate and significant genetic differentiation (Fst $=0.1098 ; \mathrm{p}<0.001 ; 1,000$ permutations) was computed between the two species. The genetic divergence and polymorphism data were used to test departures from neutrality, with both Tajima's D statistics (Tajima 1989) and the HKA test (Hudson et al. 1987) indicating no departure from neutrality.

The performed molecular analysis revealed no fixed differences between the two analysed populations. Although some exclusive synonymous and non-synonymous mutations were found, a clearer pattern of differentiation was not obtained. Therefore, we cannot infer a possible role of the obp29 gene in responses to specific pheromones or in the process of reproductive isolation. The level of genetic differentiation was not as high as that observed for other nuclear markers (Bauzer et al. 2002a, b, Bottecchia et al. 2004, Araki et al. 2009, Lins et al. 2012). This finding can be explained by the recent origin of these species within the Lu. longipalpis complex and the consequent retention of ancestral polymorphisms. Alternatively, one might also consider introgression events if reproductive barriers allowed rare events of hybridisation. Future studies expanding this analysis to other $o b p$ genes might help in the elucidation of a putative functional role of OBPs in the speciation process occurring in Lu. longipalpis s.l.

\section{REFERENCES}

Abdeladhim M, Jochim RC, Ahmed MB, Zhioua E, Chelbi I, Cherni S, Louzir H, Ribeiro JM, Valenzuela JG 2012. Updating the salivary gland transcriptome of Phlebotomus papatasi (Tunisian strain): the search for sand fly-secreted immunogenic proteins for humans. PLoS ONE 7: e47347.

Araki AS, Vigoder FM, Bauzer LG, Ferreira GE, Souza NA, Araújo IB, Hamilton JG, Brazil RP, Peixoto AA 2009. Molecular and behavioral differentiation among Brazilian populations of Lutzomyia longipalpis (Diptera: Psychodidae: Phlebotominae). PLoS Negl Trop Dis 3: e365.

Azevedo RV, Dias DB, Bretãs JA, Mazzoni CJ, Souza NA, Albano RM, Wagner G, Davila AM, Peixoto AA 2012. The transcriptome of Lutzomyia longipalpis (Diptera: Psychodidae) male reproductive organs. PLOS ONE 7: e34495.

Bauzer LGSR, Gesto JS, Souza NA, Ward RD, Hamilton JG 2002a. Molecular divergence in the period gene between two putative sympatric species of the Lutzomyia longipalpis complex. Mol Biol Evol 19: 1624-1627.

Bauzer LGSR, Souza NA, Maingon RDC, Peixoto AA 2007. Lutzomyia longipalpis in Brazil: a complex or a single species? A minireview. Mem Inst Oswaldo Cruz 102: 1-12.

Bauzer LGSR, Souza NA, Ward RD, Kyriacou CP, Peixoto AA $2002 \mathrm{~b}$. The period gene and genetic differentiation between three
Brazilian populations of Lutzomyia longipalpis. Insect Mol Biol 11: 315-323.

Bottecchia M, Oliveira SG, Bauzer LG, Souza NA, Ward RD, Garner KJ, Kyriacou CP, Peixoto AA 2004. Genetic divergence in the cacophony IVS6 intron among five Brazilian populations of $L$. longipalpis. J Mol Evol 58: 754-761.

Dias DBS 2008. Análise de genes expressos nas antenas de Lutzomyia longipalpis (Lutz e Neiva, 1912) (Diptera: Psychodidae: Phebotominae), PhD Thesis, Instituto Oswaldo Cruz, Rio de Janeiro, $243 \mathrm{pp}$.

Filatov DA 2002. ProSeq: a software for preparation and evolutionary analysis of DNA sequence data sets. Mol Ecol 2: 621-624.

González-Caballero N, Valenzuela JG, Ribeiro JM, Cuervo P, Brazil RP 2013. Transcriptome exploration of the sex pheromone gland of Lutzomyia longipalpis (Diptera: Psychodidae: Phlebotominae). Parasit Vectors 7: 56.

Hall TA 1999. BioEdit: a user-friendly biological sequence alignment editor and analysis program for Windows 95/98/NT. Nucleic Acids Symp Ser (Oxf) 41: 95-98.

Hallem EA, Carlson JR 2004. The odor coding system of Drosophila. Trends Genet 20: 453-459.

Hamilton JGC, Brazil RP, Morgan ED, Alexander B 1999a. Chemical analysis of oxygenated homosesquiterpenes: a putative sex pheromone from Lutzomyia longipalpis (Diptera: Psychodidae). Bull Entom Res 89: 139-145.

Hamilton JGC, Hooper AM, Mori K, Pickett JA, Sano S 1999b. 3-Methyl-a-himachalene confirmed and the relative stereochemistry defined, by synthesis as the sex pheromone of the sandfly Lutzomyia longipalpis from Jacobina, Brazil. Chem Commun (Camb) 4: 355-356.

Hekmat-Scafe DS, Scafe CR, Mckinney AJ, Tanouye MA 2002. Wide analysis of the odorant-binding protein gene family in $D$. melanogaster. Genome Res 12: 1357-1369.

Hudson RR, Kreitman M, Aguadé M 1987. A test of neutral molecular evolution based on nucleotide data. Genetics 116: 153-159.

Lane R, Phillips A, Molyneux DH, Ward RD 1985. Chemical analysis of the abdominal glands of two forms of Lutzomyia longipalpis: site of a possible sex pheromone? Ann Trop Med Parasitol 79: 225-229.

Li Z, Pickett JA, Field LM, Zhou JJ 2005. Identification and expression profiling of putative odorant-binding proteins in the malaria mosquitoes, Anopheles gambiae and Anopheles arabiensis. Arch Insect Biochem Physiol 58: 175-189.

Librado P, Rozas J 2009. DnaSP v.5: a software for comprehensive analysis of DNA polymorphism data. Bioinformatics 25: 1451-1452.

Lins RM, Souza NA, Brazil RP, Maingon RD, Peixoto AA 2012. Fixed differences in the paralytic gene define two lineages within the Lutzomyia longipalpis complex producing different types of courtship songs. PLoS ONE 7: e44323.

Liu R, Lehane S, He X, Lehane M, Hertz-Fowler C, Berriman M, Pickett JA, Field LM, Zhou JJ 2010. Characterisations of odorantbinding proteins in the tsetse fly Glossina morsitans morsitans. Cell Mol Life Sci 67: 919-929.

Maingon RD, Ward RD, Hamilton JG, Noyes HA, Souza N, Kemp SJ, Watts PC 2003. Genetic identification of two sibling species of Lutzomyia longipalpis (Diptera: Psychodidae) that produce distinct male sex pheromones in Sobral, Ceará state, Brazil. Mol Ecol 12: 1879-1894.

Otto TD, Vasconcellos EA, Gomes LHF, Moreira AS, Degrave WM, Mendonça-Lima L, Alves-Ferreira M 2008. ChromaPipe: a pipe- 
line for analysis, quality control and management for a DNA sequencing facility. Genet Mol Res 7: 861-871.

Pelletier J, Leal WS 2009. Genome analysis and expression patterns of odorant-binding proteins from the southern house mosquito Culex pipiens quinquefasciatus. PLoS ONE 4: e6237.

Pelosi P, Zhou JJ, Ban LP, Calvello M 2006. Soluble proteins in insect chemical communication. Cell Mol Life Sci 63: 1658-1676.

Sambrook D, Russel J 2001. Plasmids and their usefulness in molecular cloning. In Molecular cloning. A Laboratory manual, 3rd ed., Cold Spring Harbor, New York, p. 1.32-1.34.

Sirot LK, Hardstone MC, Helinski ME, Ribeiro JM, Kimura M, Deewatthanawong P, Wolfner MF, Harrington LC 2011. Towards a semen proteome of the dengue vector mosquito: protein identification and potential functions. PLoS Negl Trop Dis 5: e989.

Souza NA, Vigoder FM, Araki AS, Ward RD, Kyriacou CP, Peixoto AA 2004. Analysis of the copulatory courtship songs of Lutzomyia longipalpis in six populations from Brazil. J Med Entomol 41: 906-913.

Souza NA, Ward RD, Hamilton JG, Kyriacou CP, Peixoto AA 2002. Copulation songs in three siblings of Lutzomyia longipalpis (Diptera: Psychodidae). Trans R Soc Trop Med Hyg 96: 102-103.
Tajima F 1989. Statistical method for testing the neutral mutation hypothesis by DNA polymorphism. Genetics 123: 585-595.

Vieira FG, Sánchez-Gracia A, Rozas J 2007. Comparative genomic analysis of the odorant-binding protein family in 12 Drosophila genomes: purifying selection and birth-and-death evolution. $G e$ nome Biol 8: R235.

Ward PC, Phillips A, Burnet B, Marcondes CB 1988. The L. longipalpis complex: reproduction and distribution. In MW Service, Biosystematics of haematophagous insects, Oxford University Press, Oxford, p. 258-269.

Xu PX, Zwiebel LJ, Smith DP 2003. Identification of a distinct family of genes encoding atypical odorant-binding proteins in the malaria vector mosquito, Anopheles gambiae. Insect Mol Biol 12: $549-560$

Zhou JJ, He XL, Pickett JA, Field LM 2008. Identification of odorantbinding proteins of the yellow fever mosquito Aedes aegypti: genome annotation and comparative analyses. Insect Mol Biol 17: $147-163$

Zhou JJ, Huang W, Zhang GA, Pickett JA, Field LM 2004. "Plus-C" odorant-binding protein genes in two Drosophila species and the malaria mosquito Anopheles gambiae. Gene 327: 117-129. 